\title{
Premier cas de chronobiologie des émissions cercariennes de type infradien chez Schistosoma mansoni dans deux foyers du sud-Bénin
}

\author{
Moudachirou IBIKOUNLE ${ }^{1,4^{*}}$, Hélène MONE ${ }^{2}$, Youssouf ABOU ${ }^{4}$, \\ Dorothée KINDE-GAZARD ${ }^{3}$, Nestor G. SAKITI ${ }^{4}$, Gabriel MOUAHID $^{2}$ et \\ Achille MASSOUGBODJI ${ }^{1,3}$ \\ ${ }^{1}$ Laboratoire des Maladies Transmissibles, FAST-ISBA, Université d'Abomey-Calavi, \\ O1BP918 Cotonou, Bénin. \\ ${ }^{2}$ Laboratoire d'Ecologie et Evolution des Interactions (2EI), UMR5244 CNRS-UPVD, Université de Perpignan \\ Via Domitia, 52 Avenue Paul Alduy, 66860 Perpignan, France. \\ ${ }^{3}$ Laboratoire de Parasitologie-Mycologie, Faculté des Sciences de la Santé, Université d'Abomey-Calavi, \\ 01BP188 Cotonou, Bénin. \\ ${ }^{4}$ Département de Zoologie, Faculté des Sciences et Techniques, Université d'Abomey-Calavi, \\ 01BP526 Cotonou, Bénin. \\ *Auteur correspondant, E-mail : mibikounle2001@yahoo.fr; Tél: +2299764 5885 ; Fax : +229 21300028
}

\section{RESUME}

La schistosomose constitue au Bénin un réel problème de santé publique comme dans la plupart des autres pays africains. L'étude de la chronobiologie des émissions cercariennes horaires de deux populations de Schistosoma mansoni du sud-Bénin révèle, aussi bien en conditions de laboratoire qu'en conditions naturelles, un rythme particulier d'émission de type infradien avec 3 périodes de production : à l'aube entre 6 heures et 8 heures (Heure Locale, HL), en milieu de journée entre 09 heures et 15 ou 16 heures (HL) et le soir entre 17 heures et 20 heures (HL). Ce rythme d'émission est contraire à celui du type circadien, avec un seul pic d'émission, connu à ce jour pour les schistosomes de ce groupe. Ces deux populations de schistosomes ont été soumises à une pression sélective de même nature induisant des modifications dans le sens de l'harmonisation des processus de transmission du parasite à l'hôte vertébré. Une étude ultérieure permettra de mieux apprécier les causes de la modification du rythme circadien au type infradien.

(C) 2012 International Formulae Group. All rights reserved.

Mots Clés : Schistosoma mansoni, chronobiologie, rythme infradien, sud-Bénin.

\section{INTRODUCTION}

La schistosomose ou bilharziose est une maladie parasitaire endémique de la zone intertropicale et constitue la deuxième endémie parasitaire sur le plan mondial après le paludisme. Le nombre de personnes infectées par la bilharziose est estimé à plus de 200 millions et près de 280000 personnes décèdent chaque année des complications provoquées par l'infection (Chippaux, 2000 ; Chitsulo et al., 2004 ; WHO, 2011).

Au Bénin, deux espèces humaines de schistosomes sont signalées: Schistosoma mansoni qui sévit de façon focalisée et $S$. haematobium à distribution large avec des taux de prévalences variables d'une localité à 
une autre et selon la typologie des sites de transmission (Garba et al., 2000 ; Ibikounlé et al., 2009 ; Moné et al., 2010). Toutefois, une étude récente a révélé les zones d'hybridation naturelles entre $S$. haematobium et $S$. guineensis (Moné et al., 2012) ; ce qui laisse présager de la présence de cette dernière espèce $S$. guineensis au Bénin.

Dans l'histoire des schistosomes, le rythme des émissions des larves constitue une étape importante dans le cycle de développement des parasites. Le rôle des cercaires dans le mécanisme de transmission et le maintien du cycle ainsi que dans la dispersion du parasite est important. Il a été montré que très souvent les rythmes d'émergence des cercaires sont corrélés à une activité particulière de l'hôte à infester. Par exemple, chez les schistosomes, l'émission diurne des cercaires correspond aux heures où l'activité de l'Homme dans le milieu aquatique, en zone endémique, est maximum (Combes, 1983 ; Cassir et al., 1998 ; Thomas et al., 2007). Il existe donc une coadaptation rythme d'émission des cercaires - activité de l'hôte. Ainsi, nous distinguons à ce jour quatre grands types de rythmes à travers les «patterns » chronobiologiques des schistosomes du genre Schistosoma étudiés par différents auteurs : ceux dont le pic d'émergence est matinal ( $S$. bovis, $S$. curassoni et $S$. mattheei), correspondant généralement aux schistosomes du bétail domestique, ceux dont le pic d'émergence est tardif, crépusculaire ou nocturne $(S$. leiperi et $S$. rodhaini), correspondant aux schistosomes d'animaux sauvages : ruminants ( $S$. leiperi) et rongeurs ( $S$. rodhaini), ceux qui possèdent deux pics d'émergence, l'un au lever du jour, l'autre au crépuscule $(S$. margrebowiei), correspondant aux schistosomes des antilopes principalement et ceux dont le pic d'émergence est situé en milieu de journée ( $S$. mansoni, $S$. haematobium, $S$. guineensis et $S$. intercalatum), correspondant aux schistosomes de l'Homme (Combes et Théron, 1977 ; Mouahid et Théron, 1986 ; Mouahid et Combes, 1987; Rollinson et Southgate, 1987; Mouchet et al., 1992 ; Thomas et al., 2007). La présente étude signale, pour la première fois, la présence d'un rythme d'émission cercarienne de type infradien au niveau de deux populations de $S$. mansoni dans le sud-Bénin.

\section{MATERIEL ET METHODES Matériel}

L'étude de la dynamique d'émission cercarienne horaire est réalisée sur deux populations de Schistosoma mansoni provenant respectivement du foyer de TohoTodougba $\left(2^{\circ} 12^{\prime} 20.09^{\prime \prime} \mathrm{E} ; 6^{\circ} 23^{\prime} 23.56^{\prime \prime} \mathrm{N}\right)$ et de celui de Kpinnou (146'44.13"E; $6^{\circ} 28^{\prime} 41.11^{\prime \prime N}$ ) au sud du Bénin. La population de parasite de Toho-Todougba est extraite à partir de fèces de 4 pêcheurs et celle de Kpinnou à partir de fèces de 4 écoliers (Ibikounlé et al., 2009). Deux populations locales de Biomphalaria pfeifferi provenant de Toho-Todougba et Kpinnou sont utilisées. Le nombre de mollusques utilisés par expérience a varié de 6 à 9 et le nombre de jours d'expérience a varié de 6 à 9 jours. Le Tableau 1 présente les différentes combinaisons hôte-parasite étudiées.

\section{Méthodes d'étude}

Pour chaque population de schistosomes, l'étude de la chronobiologie des émissions cercariennes horaires est réalisée dans les conditions expérimentales dans le Laboratoire d'Ecologie et Evolution des Interactions (2EI), UMR5244 CNRS-UPVD du Centre de Biologie et d'Ecologie Tropicale et Méditerranéenne (CBETM) à Perpignan, en France et dans les conditions naturelles au Bénin.

$\mathrm{Au}$ laboratoire, l'infestation des mollusques est monomiracidiale (Tableau 1). Chaque mollusque est exposé à un seul miracidium issu de l'éclosion des œufs de $S$. mansoni prélevés des fèces des patients. Après quatre semaines post-infestation, les mollusques sont testés et les positifs sont placés à l'étuve, en photopériodicité équilibrée $12: 12$. La température dans l'étude est maintenue constante à $25^{\circ} \mathrm{C}$ et l'éclairage est assuré par une lampe électrique. Le jour apparaît de façon brusque à 6,00 heures et la nuit apparaît aussi brusquement à 18,00 
Tableau 1: Répartition des différents couples hôte-parasite étudiés.

\begin{tabular}{|c|c|c|c|c|c|}
\hline $\begin{array}{l}\begin{array}{l}\text { Population } \\
\text { parasites }\end{array} \\
\end{array}$ & $\begin{array}{c}\text { Conditions } \\
\text { expérimentales }\end{array}$ & Population mollusques & $\begin{array}{c}\text { Nombre de } \\
\text { miracidiums }\end{array}$ & $\mathrm{Nm}$ & $\mathbf{N j}$ \\
\hline \multirow[t]{2}{*}{ Kpinnou } & Laboratoire & $\begin{array}{l}\text { B. pfeifferi de } \\
\text { Kpinnou }\end{array}$ & 1 & 3 & 9 \\
\hline & Naturelle & $\begin{array}{c}\text { B. pfeifferi de } \\
\text { Kpinnou }\end{array}$ & 1 & 7 & 6 \\
\hline \multirow{2}{*}{$\begin{array}{l}\text { Toho- } \\
\text { Todougba }\end{array}$} & Laboratoire & $\begin{array}{c}\text { B. pfeifferi de } \\
\text { Toho-Todougba }\end{array}$ & 2 & 6 & 9 \\
\hline & Naturelle & $\begin{array}{l}\text { B. pfeifferi de } \\
\text { Toho-Todougba }\end{array}$ & 1 & 9 & 6 \\
\hline
\end{tabular}

heures. Chaque mollusque positif est placé dans un verre en plastique, transparent, étiqueté et contenant environ $150 \mathrm{ml}$ d'eau de forage à température. A chaque heure, les mollusques sont transférés dans des récipients identiques portant la même étiquette et contenant le même volume d'eau de forage à la même température ainsi que de la nourriture. Les échantillons, ainsi prélevés, sont filtrés individuellement sur un filtre polyamide Nitrel $(25 \mu \mathrm{m}$ de vide de maille). Les cercaires retenues sur le filtre sont colorées avec une solution de Lugol puis comptées sous la loupe binoculaire.

En condition naturelle, l'expérience est réalisée dans une enceinte de $4 \mathrm{~m}$ sur $4 \mathrm{~m}$ naturellement éclairée (absence d'électricité) et thermorégulée (climatisée). Le jour se fait entre 6 heures et 7 heures et la nuit tombe entre 18 heures et 19 heures. La température expérimentale varie entre $23{ }^{\circ} \mathrm{C}$ le matin et 25 ${ }^{\circ} \mathrm{C}$ la journée et la nuit. Les mollusques utilisés dans l'expérience en conditions naturelles au Bénin ont été infestés au CBETM de Perpignan, puis transportés au Bénin. Pour ce faire, nous avons observé pour les mollusques parasités une phase de repos de 72 heures au Bénin, afin d'éliminer toute perturbation qui pourrait influencer le rythme normal d'émission cercarienne. Après 72 heures de conditionnement dans l'enceinte, chaque mollusque est également placé dans un verre en plastique, transparent, étiqueté et contenant environ $150 \mathrm{ml}$ d'eau de puits à la température ordinaire. L'ensemble est placé à proximité de la partie vitrée de l'enceinte plus éclairée à la lumière du jour et subi le même traitement que précédemment. Dans les deux conditions, les mollusques sont nourris avec des feuilles de laitue fraîches. Pendant la durée de chaque expérience, lorsqu'un mollusque meurt, il est remplacé par un autre mollusque, de taille approximativement égale.

\section{Analyses statistiques}

La comparaison des données chronobiologiques horaires des trois phases (aube, journée et crépuscule) s'est faite au moyen de test de comparaison de moyenne (test T de Student) sur le logiciel StatView ${ }^{\circledR}$ et le Z-Test. Les différences sont considérées comme significatives au seuil de 5\%. Les moyennes horaires ainsi que les écarts standards et les graphiques ont été réalisés à l'aide du logiciel Excel $2007^{\circledR}$ de Microsoft $^{\circledR}$ Office.

\section{RESULTATS}

Chronobiologie en conditions de laboratoire

- S. mansoni de Kpinnou: L'étude (Figure 1A) est faite sur 3 B. pfeifferi de Kpinnou parasités, chacun avec 1 miracidium de $S$. mansoni local et l'expérience a duré 9 jours (Nombre de cycles $=27$ ). La première émission de cercaires est observée à 6 heures et la dernière à 18 heures avant la phase obscure. On distingue trois phases dans le profil moyen d'émission dont la première à l'aube et les deux autres principalement 
diurnes. Bien qu'étalée sur toute la journée de 6 heures à 18 heures, la production cercarienne est concentrée sur trois temps : de 6 heures à 8 heures; avec 12,02\% de la production journalière moyenne, de 9 heures à 16 heures avec $84,70 \%$ et de 17 heures à 18 heures, représentant $3,28 \%$ de la production journalière moyenne. L'étude de la chronobiologie montre trois pics individualisés $(\mathrm{p}<0,05)$. Le premier pic se situe au petit matin entre 6 heures et 7 heures et représente $9,48 \% \pm 2,63$ de la production cercarienne moyenne, le deuxième pic se situe entre 11 heures et 12 heures et représente $39,84 \% \pm 2,57$ de la production moyenne et le troisième pic se situe entre 16 heures et 17 heures et représente $2,16 \% \pm 0,62$ de la production moyenne.

- S. mansoni de Toho-Todougba: La Figure 2A est représentée à partir de la production cercarienne de 6 mollusques exposé chacun avec 2 miracidiums de parasite local et l'expérience a durée 9 jours (soit un nombre de cycles égal à 54). La première émission cercarienne est observée à 6 heures et la dernière à $19 \mathrm{~h}$. La production cercarienne est étalée sur toute la journée. Toutefois, nous pouvons distinguer une production cercarienne concentrée sur trois temps $(\mathrm{p}<0,05)$ : de 6 heures à 8 heures avec $6,32 \%$ de la production journalière moyenne, de 9 heures à 18 heures avec 93,61\% et une faible production en début de la scotophase à 19 heures, représentant $0,07 \%$ de la production journalière moyenne. L'étude de la chronobiologie montre deux pics individualisés. Le premier pic se situe au matin entre 6 heures et 7 heures et représente $5,38 \% \pm 1,36$ de la production cercarienne moyenne et le deuxième pic se situe entre 10 heures et 11 heures et représente $38,56 \% \pm$ 1,79 de la production moyenne.

\section{Chronobiologie en conditions naturelles}

- S. mansoni de Kpinnou: La chronobiologie (Figure 2A) est obtenue à partir d'une population locale de 7 B. pfeifferi parasités chacun avec 1 miracidium. L'expérience a duré 6 jours, soit 42 cycles. La première émission de cercaires est observée à
6 heures et la dernière à 20 heures. On distingue trois phases dans le profil moyen de cette émission dont deux sont principalement diurnes et la troisième s'étendant jusqu'à la nuit (20 heures). On distingue trois temps de production $(\mathrm{p}<0,05)$ : de 6 heures à 9 heures avec $25,71 \%$ de la production journalière moyenne, de 10 heures à 15 heures avec $63,90 \%$ et de 16 heures à 20 heures, représentant $10,39 \%$ de la production journalière moyenne. L'étude de la chronobiologie montre trois pics individualisés. Le premier pic se situe au petit matin entre 7 heures et 8 heures et représente $11,69 \% \pm 1,01$ de la production cercarienne moyenne; le deuxième pic se situe entre 11 heures et 12 heures et représente $31,14 \% \pm$ 1,36 de la production moyenne et le troisième pic se situe entre 18 heures et 19 heures et représente $6,66 \% \pm 0,93$ de la production moyenne.

- S. mansoni de Toho-Todougba: La Figure (2B) est produite à partir de 9 mollusques parasités avec 1 miracidium et le nombre de jours est de 6 . Le nombre de cycles est alors $\mathrm{N}=54$. La première émission de cercaires est observée à 6 heures à l'aube et la dernière à 20 heures en obscurité. Nous avons un profil moyen d'émission composé de trois principales phases dont deux entièrement diurnes et une troisième phase partagée entre le jour et la nuit. La production cercarienne est concentrée sur trois temps de la journée $(\mathrm{p}<0,05)$ : de 6 heures à 8 heures avec $24,71 \%$ de la production journalière moyenne, de 9 heures à 16 heures avec une production journalière moyenne de $64,14 \%$ et de 17 heures à 20 heures avec une production journalière moyenne de $11,15 \%$. L'étude de la chronobiologie montre trois pics individualisés. Le premier pic se situe à l'aube entre 6 heures et 7 heures et représente $14,84 \% \pm 1,40$ de la production cercarienne moyenne; le deuxième pic se situe au milieu $\mathrm{du}$ jour, entre 11 heures et 12 heures et représente $31,49 \% \pm 2,17$ de la production moyenne et le troisième pic se situe au crépuscule, entre 18 heures et 19 heures et représente $6,92 \% \pm 0,17$ de la production moyenne. 


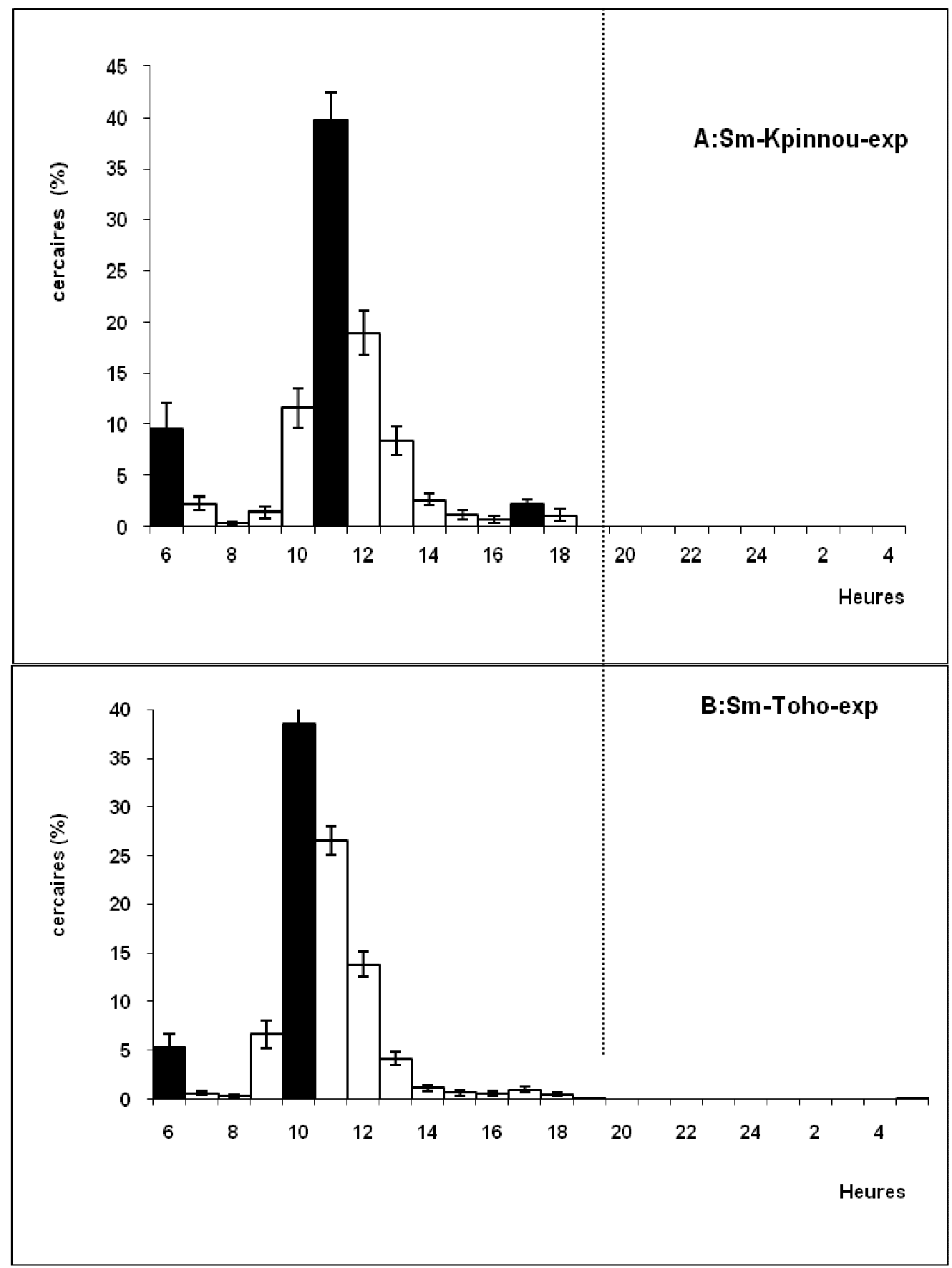

Figure 1: Emission cercarienne horaire de deux populations de S. mansoni du sud-Bénin (A : Kpinnou et B: Toho-Todougba, respectivement) en conditions de laboratoire exprimée en pourcentage de la production journalière totale. Les colonnes sombres indiquent les pics d'émission ( $p<0,05)$, les barres représentent l'écart standard. 


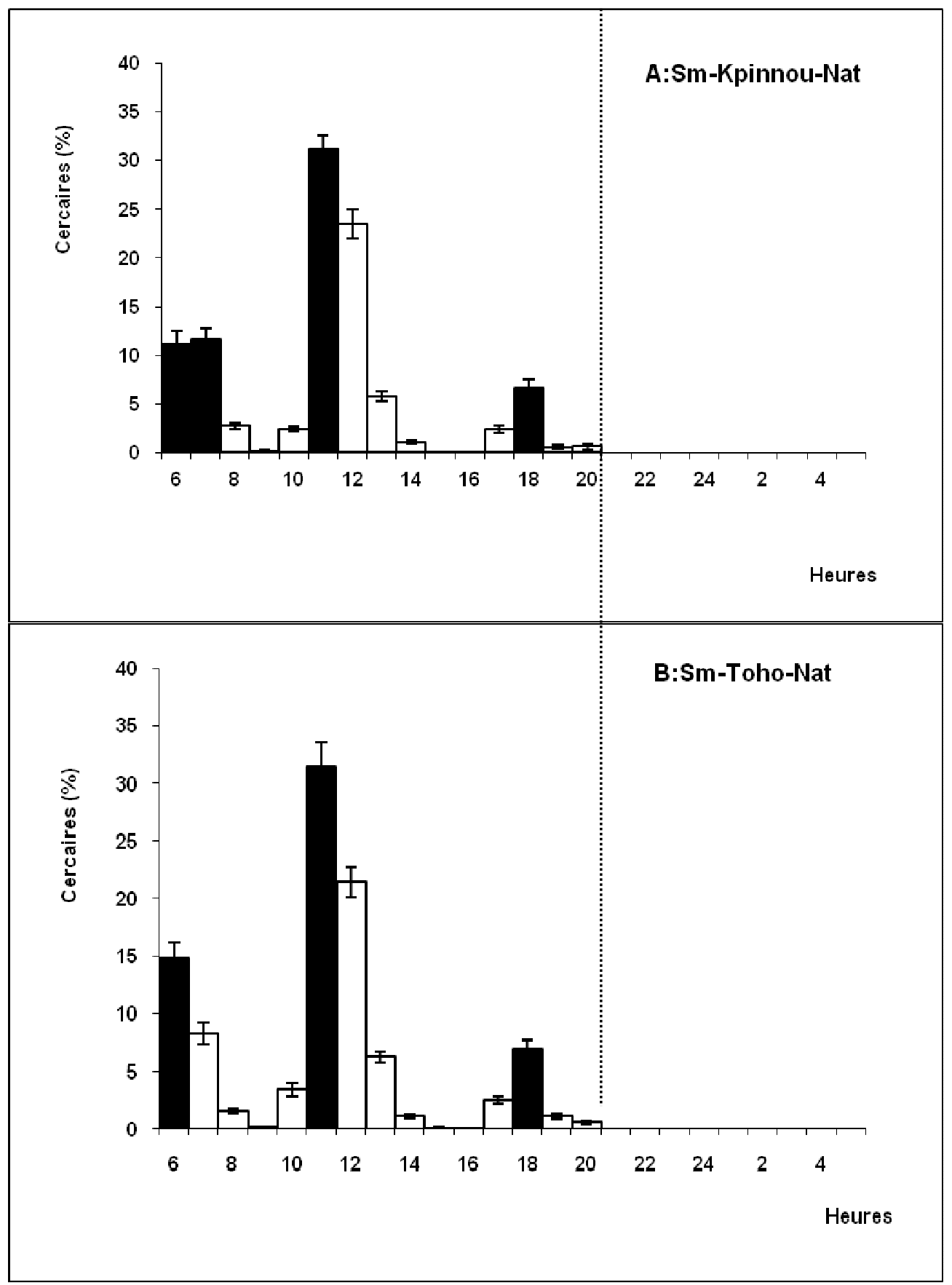

Figure 2 : Emission cercarienne horaire de deux populations de $S$. mansoni du sud-Bénin (A : Kpinnou et B : Toho-Todougba, respectivement) en conditions naturelles exprimée en pourcentage de la production journalière totale. Les colonnes sombres indiquent les pics d'émission $(\mathrm{p}<0,05)$, les barres représentent l'écart standard. 


\section{DISCUSSION}

L'étude de l'émission cercarienne horaire des deux populations de Schistosoma mansoni du sud-Bénin donne, d'une façon générale, un rythme d'émission cercarienne de type infradien. Pour les deux populations de $S$. mansoni, on distingue trois phases de production avec trois pics d'émission cercarienne: la première phase au cours de laquelle le quart de la production journalière moyenne est réalisé, a lieu à l'aube avec un pic d'émission entre 6 heures et 9 heures et représentant de 5,38 à $14,84 \%$ de la production cercarienne journalière moyenne, la deuxième phase, plus grande, au cours de laquelle plus de la moitié de la production journalière moyenne est libérée, a lieu en milieu de journée, avec un pic d'émission entre 11 heures et 12 heures représentant de 31,14 à $39,84 \%$ de la production cercarienne journalière moyenne, et le troisième temps de production a lieu en scotophase avec un pic entre 18 heures et 19 heures et représente de 2,16 à $6,92 \%$ de la production cercarienne journalière moyenne. Il existe donc pour les deux populations de schistosomes trois temps de production cercarienne avec trois pics d'émission bien individualisés; ce qui est contraire à l'émission cercarienne à un seul pic connu pour l'espèce $S$. mansoni.

Dans la mesure où le rythme infradien (3 pics d'émission de cercaires) est obtenu aussi bien en conditions de laboratoire qu'en conditions naturelles, l'influence possible des conditions expérimentales sur l'émergence des larves n'est pas envisageable parmi les hypothèses explicatives du mécanisme chronobiologique des deux populations de schistosomes étudiées.

Dans la littérature, les études expérimentales ont montré que dans le cas d'une double infestation d'un mollusque par deux espèces de schistosomes appartenant au même groupe (Rollinson et Southgate, 1987), l'expression chronobiologique est individuelle comme cela fut observer avec Biomphalaria glabrata doublement infecté par S. mansoni à émission diurne et $S$. rodhaini à émission nocturne (Northon et al., 2008 ; Steinauer et al., 2008). Cette hypothèse ne paraît pas plausible pour expliquer l'apparition de trois temps d'émission d'une part parce qu'il s'agit des souches humaines de parasite et d'autre part parce que les mollusques utilisés ont été infestés par 1 ou 2 miracidiums issus des œufs récoltés dans les selles humaines.

Des cas particuliers de rythme d'émergence des cercaires ont été signalés chez certaines espèces humaines de schistosomes. C'est le cas en Thaïlande, avec S. mekongi, qui est un parasite essentiellement de l'Homme mais qui possède un pic d'émergence très matinal (6 heures à 8 heures) (Théron et Mingyi, 1986). C'est aussi le cas chez une population de $S$. japonicum qui possède une chronobiologie nocturne (pic d'émergence entre 18 heures et 20 heures). Ces auteurs lient le comportement de ces parasites à une adaptation à l'éthologie de leurs hôtes vertébrés. En effet, en Thaïlande et au Laos, les travaux dans les rizières se font très tôt le matin et en Philippines, $S$. japonicum serait adaptée à des hôtes de la faune sauvage dont l'activité serait plutôt crépusculaire ou nocturne ( $\mathrm{Lu}$ et al., 2009). Les deux populations de $S$. mansoni du Bénin se seraient adaptées aux rythmes d'activités des populations humaines riveraines.

Nos résultats montrent également une homogénéité chronobiologique des émissions cercariennes horaires au niveau intraspécifique. Cette harmonie de rythmes serait due à des conditions de transmission identiques dans les différentes localités, confirmant l'hypothèse que les phénomènes chronobiologiques possèdent une base génétique (Théron et Combes, 1988 ; Cassir et al., 1998 ; Thomas et al., 2007). Les deux populations de $S$. mansoni présentent entre elles un profil d'émission très proche, ce qui prouve qu'elles ont été soumises à une pression sélective de même nature induisant des modifications dans le sens de l'harmonisation des processus de transmission du parasite à l'hôte vertébré. Les études ont montré des variétés chronobiologiques différentes aussi bien chez $S$. haematobium en Afrique de l'Ouest (N'Goran et al., 1997) que 
chez $S$. mansoni des foyers antillais (Théron et al., 1997) mais c'est pour la première fois qu'il est présenté un rythme infradien chez $S$. mansoni. A notre avis, trois hypothèses inclusives peuvent justifier ces différences : (1) l'écologie et les comportements sociaux des hôtes définitifs (Homme) de ces régions, (2) des phénomènes d'introgression d'une espèce par une autre espèce comme cela a été montré entre $S$. bovis et $S$. haematobium au Niger (Brémond et al., 1993) et entre $S$. haematobium et $S$. guineensis au Bénin (Moné et al., 2012) et (3) la participation des hôtes réservoirs (rongeurs et singe) dans le cycle de transmission de la parasitose comme prouvé dans les foyers guadeloupéens et sénégalais (Théron et al., 1992, Sène et al., 1996 ; Alarcón de Noya et al., 1997). Les études ultérieures basées sur la dynamique horaire des activités des différents hôtes vertébrés (Homme, singe et rongeurs) permettront de mieux apprécier les fondements génétiques de modification des émissions larvaires de ces parasites.

\section{Conclusion}

Les deux populations de S. mansoni concernées présentent globalement un rythme infradien avec trois temps d'émission cercarienne : à l'aube, autour de midi et au crépuscule. Il s'agirait d'une stratégie de parasite pour se maintenir dans le milieu en maximisant le rendez-vous ou temps de contact avec son ou ses hôtes vertébrés.

\section{REMERCIEMENTS}

Cette étude a été entièrement financée par le Ministère Français des Affaires Etrangères et Européennes à travers le projet CORUS-2-6069 intitulé «épidémiologie et écologie des schistosomoses au Bénin ».

\section{REFERENCES}

Alarcón de Noya B, Pointier JP, Colmenares C, Théron A, Balzan C, Cesari IM, González S, Noya O. 1997. Natural Schistosoma mansoni infection in wild rats from Guadeloupe: parasitological and immunological aspects. Acta Tropica, 68: 11-21.

Brémond P, Sellin E, Nameaua B, Labbo R, Théron A, Combes C. 1993. Arguments en faveur d'une modification du genome (introgression) du parasite humain Schistosoma haematobium par des gènes de S. bovis, au Niger. Comptes Rendus de l'Académie des Sciences, 316: 667-670.

Cassir P, Brugerolle G, Combes C, Grain J, Raibaut A. 1998. Le Parasitisme, un Equilibre Dynamique. Masson: Paris.

Chippaux JP. 2000. Control of schistomiasis : realities and futurology. Médecine Tropicale, 60: 54-55.

Chitsulo L, Loverde P, Engels D. 2004. Schistosomiasis. Nat. Rev. Microbiol., 2: 12-13.

Combes C. 1983. Les parasites et leurs cibles vivantes. Pour la Science, 70: 64-72.

Combes C, Théron A. 1977. Rythmes d'émergence des cercaires de Trématodes et leur intérêt dans l'infestation de l'homme et des animaux. Instituto de Biologia Publicaciones, 4: 141-150.

Garba A, Kinde-Gazard D, Makoutodé M, Boyer N, Ernould JC, Chippaux JP, Massougbodji A. 2000. Preliminary evaluation of morbidity due to $S$. Haematobium and S. Mansoni in the area of the future Adjarala Dam in Benin. Santé, 10: 323-328.

Ibikounlé M, Mouahid G, Sakiti NG, Massougbodji A, Moné H. 2009. Freshwater snail diversity in Benin (West Africa) with a focus on human schistosomiasis. Acta Tropica, 111: 2934.

Lu DB, Wang TP, Rudge JW, Donnelly CA, Fang GR, Webster JP. 2009. Evolution in a multi-host parasite: Chronobiological circadian rhythm and population genetics of Schistosoma japonicum cercariae indicates contrasting definitive host reservoirs by habitat. Int. J. Parasitol., 39: 1581-1588.

Moné H, Ibikounlé M, Massougbodji A, Mouahid G. 2010. Human schistosomiasis in the Economic 
Community of West African States (ECOWAS): epidemiology and control. Advances in Parasitology, 71: 33-91.

Moné H, Minguez S, Ibikounlé M, Allienne JF, MassougbodjiA, Mouahid G. 2012. Natural interactions between $S$. haematobium and $S$. guineensis in the Republic of Benin. The Scientific World Journal. Article ID 793420, 8 pages, doi:10.1100/2012/793420.

Mouahid A, Combes C. 1987. Genetic variability of Schistosoma bovis cercarial production according to miracidial dose. Journal of Helminthology, 61: 89-94.

Mouahid A, Théron A. 1986. Schistosoma bovis: variability of cercarial production as related to the snail hosts: Bulinus truncatus, B. wrighti and Planorbarius metidjensis. Int. J. Parasitol., 17: 14311434.

Mouchet F, Théron A, Brémond P, Sellin E, Sellin B. 1992. Pattern of cercarial emergence of Schistosoma curassoni from Niger and comparison with three sympatric species of schistosomes. Journal of Parasitology, 8: 61-63.

N'Goran EK, Brémond P, Sellin E, Sellin B, Théron A. 1997. Intraspecific diversity of Schistosoma haematobium in West Africa : chronobiology of cercarial emergence. Acta Tropica, 66: 35-44.

Norton A, Rollinson D, Richards L, Webster J. 2008. Simultaneous infection of Schistosoma mansoni and S. Rodhaini in Biomphalaria glabrata: impact on chronobiology and cercarial behaviour. Parasit. Vect., 43: 1-9.

OMS. 2011. Relevé épidémiologique hebdomadaire, 86e année, 9(86). OMS; 73-80.

Rollinson D, Southgate VR. 1987. The genus Schistosoma: a taxonomic appraisal. In The Biology of Schistosomes: from Genes to Latrines, Rollinson D, Simpson AJG (eds). Academic Press: London; 1-49.

Sène M, Duplantier JM, Marchand B, Hervé JP. 1996. Susceptibility of rodents to infection with Schistosoma mansoni in Richard-Toll (Senegal). Parasite, 3: 321326.

Steinauer ML, Mwangi IN, Maina GM, Kinuthia JM, Mutuku MW, Agola EL, Mungai B, Mkoji GM, Loker ES. 2008. Interactions between natural populations of Human and Rodent schistosomes in the Lake Victoria Region of Kenya: A Molecular epidemiological approach. Plos. Neg. Dis., 2: e222.

Théron A, Mingyi X. 1986. Rythme d'émission des cercaires de Schistosoma japonicum de Chine continentale par des Oncomelania hupensis. Annal. Parasitol. Hum. Comp., 61: 553-558.

Théron A, Combes C. 1988. Genetic analysis of cercarial emergence rhythms of Schistosoma mansoni. Behavioral Geneic, 18: 201-209.

Théron A, Pointier JP, Morand S, ImbertEstable D, Borel G. 1992. Long-term dynamics of natural populations of Schistosoma mansoni among Rattus rattus in patchy environment. Parasitology, 104: 291-298.

Théron A, Mouahid G, Moné H. 1997. Schistosoma mansoni: cercarial shedding patterns from a mixed infection of Biomphalaria glabrata with two (early and late) chronobiological variants. Parasitology Research, 83: 356-358.

Thomas F, Guégan JF, Renaud F. 2007. Ecologie et Evolution des Systèmes Parasites. De Boeck: Paris. 